\title{
Therapeutic drug monitoring of amlodipine and the Z-FHL/HHL ratio: Adherence tools in patients referred for apparent treatment-resistant hypertension
}

\author{
E S W Jones, ${ }^{1} \mathrm{MD}$, PhD; M Lesosky, ${ }^{1} \mathrm{PhD} ;$ M Blockman, ${ }^{1} \mathrm{MD} ;$ S Castel,,${ }^{1} \mathrm{PhD} ; \mathbf{E}$ H Decloedt, ${ }^{2} \mathrm{MD} ; \mathrm{S}$ L U Schwager, ${ }^{3} \mathrm{MSc} ;$ \\ E D Sturrock, ${ }^{3} \mathrm{PhD} ; \mathrm{L}$ Wiesner, ${ }^{1} \mathrm{PhD} ; \mathbf{B} \mathrm{L}$ Rayner, ${ }^{1} \mathrm{MD}, \mathrm{PhD}$ \\ ${ }^{1}$ Department of Medicine, Groote Schuur Hospital and Faculty of Health Sciences, University of Cape Town, South Africa \\ ${ }^{2}$ Department of Medicine, Tygerberg Hospital and Faculty of Medicine and Health Sciences, Stellenbosch University, Cape Town, South Africa \\ ${ }^{3}$ Department of Integrative Biomedical Sciences, Groote Schuur Hospital and Faculty of Health Sciences, University of Cape Town, South Africa
}

Corresponding author: E S W Jones (eswjones@gmail.com)

\begin{abstract}
Background. Non-adherence to antihypertensives is a cause of 'pseudo-treatment-resistant' hypertension.
Objective. To determine whether monitoring plasma amlodipine concentrations and inhibition of angiotensin-converting enzyme (ACE) can be adjunct adherence tools.

Methods. Patients with hypertension who were prescribed enalapril and amlodipine were enrolled. Blood pressures (BPs) were monitored and an adherence questionnaire was completed. Steady-state amlodipine was assayed using liquid chromatography-mass spectrometry and degree of ACE inhibition using the Z-FHL/HHL (z-phenylalanine-histidine-leucine/hippuryl-histidine-leucine) ratio.

Results. One hundred patients (mean (standard deviation) age 50.5 (12) years, $46 \%$ male) were enrolled. Based on plasma assays, $26 / 97$ patients $(26.8 \%)$ were unsuppressed by enalapril and 20/100 (20\%) were sub-therapeutic for amlodipine. There were significant BP differences based on plasma levels of the medication: 21/20 mmHg lower in the group with suppressed ACE and 26/20 mmHg in the group with steady-state amlodipine concentrations.

Conclusions. Monitoring antihypertensive adherence by assaying plasma medication concentrations is a feasible option for evaluating true v. pseudo-resistant hypertension.
\end{abstract}

S Afr Med J 2017;107(10):887-891. DOI:10.7196/SAMJ.2017.v107i10.12268

Globally, high blood pressure (BP) is the leading single risk factor for cardiovascular disease, and the prevalence continues to escalate. ${ }^{[1,2]}$ Hypertension is an independent risk factor for peripheral vascular disease, heart failure, coronary artery disease, stroke and endstage renal disease. ${ }^{[3]}$ According to National Health and Nutrition Examination Survey data, the prevalence of resistant hypertension is $12.8 \%$ of treated subjects in the USA. ${ }^{[4]} \mathrm{A}$ patient's usual BP is 'strongly and directly' associated with cardiovascular and overall mortality, and lowering the BP reduces this risk. ${ }^{[5]}$ Despite the unquestionable benefits of treating hypertension, consistent control of $\mathrm{BP}$ is an elusive goal. Although control of BP varies widely in different countries, a consistent finding is that $\sim 50 \%$ of treated patients fail to achieve a goal of $<140 / 90 \mathrm{mmHg}{ }^{[6]}$ Long-term adherence is a key factor associated with BP control, ${ }^{[7]}$ and lack of adherence is associated with increased all-cause mortality in hypertensive patients. ${ }^{[8]}$ In a longitudinal study of antihypertensive dosing histories of 4783 patients in 21 phase IV clinical studies where patients were prescribed once-a-day antihypertensive treatment, about half of the patients had stopped taking their treatment within 1 year, and in many there was poor execution of treatment. ${ }^{[9]}$ Furthermore, in a cross-sectional analysis of 2180 patients in Louisiana, USA, 50\% had low to medium adherence to antihypertensives, associated with an increased risk of uncontrolled hypertension. ${ }^{[10]}$ Reasons cited for non-adherence were lack of access to medication, inadequate patient self-issuing of medication and poor patient-doctor interaction.

Monitoring antihypertensive therapy adherence is essential to define whether patients are truly treatment resistant and to avoid unnecessary special investigations and medication costs in response to poor BP control. Adherence assessment is challenging. Methods that have been used are self-reported adherence, pill counting, telephonic questioning, monitoring electronic pharmacy refill records and pharmacist monitoring. Each of these methods is limited by the fact that they do not measure whether the patient actually ingested the medication.

Therapeutic drug monitoring (TDM) is frequently employed in chronic diseases such as epilepsy and HIV to optimise medication dosing. During TDM a steady-state medication plasma concentration, at a particular time point, is measured and compared with a known therapeutic range. This range consists of an upper toxic concentration and a lower sub-therapeutic concentration. Following TDM, medication dosing may be adjusted to optimise efficacy and minimise toxicity. In practice, TDM is frequently used as a measure of adherence. ${ }^{[1]}$ TDM of antiepileptic medications has revealed that low plasma concentrations predict the risk of status epilepticus. ${ }^{[12]}$ Assays have been developed to determine adherence in clinical trials, ${ }^{[13]}$ but this has not been applied in clinical practice.

The usefulness of TDM as an adherence measure is limited by inferences about long-term adherence. Medications with a short half-life do not reach steady state, and plasma concentrations only reflect adherence to the last dose taken. TDM monitoring is most useful in medications where there is a correlation between plasma concentrations and clinical effect or toxicity and those with a narrow therapeutic index. Amlodipine, a calcium channel blocker used as a first-line antihypertensive agent, has a long elimination half-life of 
$\sim 60$ hours. ${ }^{[14]}$ Furthermore, amlodipine steady-state trough plasma concentrations at different doses have been established (Table 1) and are related to clinically relevant BP lowering. These factors make amlodipine ideal for TDM to measure adherence.

The Z-FHL/HHL (z-phenylalanine-histidine-leucine/hippurylhistidine-leucine) ratio is a measure of the inhibition of serum angiotensin-converting enzyme (ACE) by ACE inhibitors such as enalapril, the commonly used ACE inhibitor in South Africa. Lack of inhibition of serum ACE as determined by the Z-FHL/HHL ratio is considered a measure of the lack of ACE inhibition. ${ }^{[18]}$

According to the 2014 South African Hypertension Guideline, calcium channel blockers, including amlodipine, and ACE inhibitors, such as enalapril, are the cornerstones of antihypertensive therapy in conjunction with thiazide diuretics. ${ }^{[19]}$

\section{Objective}

To determine whether a random steady-state plasma amlodipine concentration and Z-FHL/HHL ratio in the clinic setting could independently indicate adherence to medication for BP control. Owing to the long half-life of amlodipine, it is unlikely that occasional missed doses would be detected with amlodipine plasma concentrations. However, it is likely that this method would detect clinically relevant chronic non-adherence to amlodipine. The Z-FHL/ HHL ratio would assist in detecting missed doses of enalapril.

Trough amlodipine plasma concentrations were used in conjunction with the Z-FHL/HHL ratio to help determine both short- and long-term patient non-adherence.

\section{Methods}

Patients attending a referral hypertension clinic for apparent treatment-resistant hypertension, who were receiving both enalapril and amlodipine with additional antihypertensives, were enrolled into the study. After providing written informed consent, patients underwent BP monitoring and completed a questionnaire relating to administration of medication, use of pill boxes, dosing schedule, names of the medications, dosage and omission of medication. Trough blood samples were taken for amlodipine plasma concentrations and the Z-FHL/HHL ratio as a measure of inhibition of ACE. The same assessments were repeated at the next follow-up visit.

The University of Cape Town Faculty of Health Sciences Human Research Ethics Committee approved the study (ref. no. 169/2013).

\section{BP measurement and adherence questionnaire}

$\mathrm{BP}$ was measured in the seated position at 2-minute intervals after 5 minutes' rest, using an ambulatory BP monitor (Spacelabs Healthcare, USA) with an appropriate-sized cuff. The means of six BPs were used to assess BP control. Patients were questioned about their knowledge of the dose, timing and names of their medications. They were asked who issued the medications, whether a pill box was used, and to indicate how adherent they were with the medications.

\footnotetext{
Amlodipine assay

Plasma amlodipine concentrations were determined using a validated liquid chromatography-tandem mass spectrometry assay developed in the Division of Clinical Pharmacology, University of Cape Town. The assay was validated over the concentration range $0.117-30 \mathrm{ng} /$ $\mathrm{mL}$. A concentration of $<2.5 \mathrm{ng} / \mathrm{mL}$ for amlodipine was considered undetectable and $>7 \mathrm{ng} / \mathrm{mL}$ was considered steady state. Values between 2.5 and $7 \mathrm{ng} / \mathrm{mL}$ were indeterminate. ${ }^{[14]}$

The trough blood samples were processed with a protein precipitation extraction method using $100 \mu \mathrm{L}$ plasma with $200 \mu \mathrm{L}$
}

precipitation solution (acetonitrile) containing a stable isotopelabelled internal standard, amlodipine-d4, at a concentration of $1 \mathrm{ng} / \mathrm{mL}$.

Isocratic chromatographic separation was achieved on a Luna C18 $5 \mu \mathrm{m} \operatorname{PFP}(2) 5 \times 2.0 \mathrm{~mm}$ analytical column (Phenomenex, USA). The mobile phase consisted of $10 \mathrm{mM}$ ammonium formate and acetonitrile $(20: 80, \mathrm{v} / \mathrm{v})$ and was delivered at a flow rate of $400 \mu \mathrm{L} / \mathrm{min}$.

An AB Sciex API 5500 Q Trap mass spectrometer (Sciex, USA) was operated at unit resolution in the multiple reaction monitoring mode, monitoring the transition of the protonated molecular ion $\mathrm{m} / \mathrm{z} 409.0$ to the product ions at $\mathrm{m} / \mathrm{z} 238.0$ for amlodipine, and monitoring the protonated molecular ions at $\mathrm{m} / \mathrm{z} 413.1$ to the product ions at $\mathrm{m} / \mathrm{z} 298.0$ for amlodipine-d4.

The combined accuracy and precision (expressed as the percent coefficient of variation) statistics of the quality controls during interbatch validation $(N=18$; high, medium and low) were $110.2 \%, 112.2 \%$ and $110.3 \%$ and $4.2 \%, 3.6 \%$ and $4.2 \%$, respectively.

\section{Z-FHL/HHL ratio}

ACE activity was measured by the hydrolysis of two synthetic substrates (Z-FHL and HHL) in a sensitive fluorimetric assay, as described previously. ${ }^{[20]}$ The ratio of enzyme activities obtained with substrates Z-FHL and HHL provides a measure of ACE inhibitor present in plasma and can be used to detect the presence of any ACE inhibitor ${ }^{[18]}$

Heparinised plasma $(10 \mathrm{~mL})$ diluted $1 / 5$ in phosphate-buffered saline was incubated with $30 \mathrm{~mL}$ of substrate $(2 \mathrm{mM}$ for Z-FHL and $5.7 \mathrm{mM}$ for $\mathrm{HHL}$ ) in $0.1 \mathrm{M}$ potassium phosphate, $0.3 \mathrm{M} \mathrm{NaCl}$ and $10 \mathrm{mM} \mathrm{ZnSO}_{4}, \mathrm{pH} 8.3$, at $37^{\circ} \mathrm{C}$ for 1 hour or 5 hours. Plasma samples with ACE activity $>15 \mathrm{mU} / \mathrm{mL}$ using HHL were incubated for 1 hour. A Z-FHL/HHL ratio of $<1.75$ was considered to indicate nonadherence to ACE inhibitors. Reagents were purchased from Merck and Sigma-Aldrich (Germany), and benzyloxycarbonyl L-Phe-L-HisLeu (Z-FHL) from Bachem AG (Switzerland).

\section{Statistical analysis}

Descriptive summaries were calculated at baseline using frequency (percentages) or medians with interquartile ranges (IQRs). ACE- and amlodipine-related values were summarised with means (standard deviations (SDs)). Thresholds for suppression v. non-suppression (ACE) and for steady state v. undetectable v. indeterminate (amlodipine) were based on available literature and inspection of ACE and amlodipine values. Fisher's exact test was used to compare proportions of patients who had controlled v. uncontrolled BPs between groups, and the Wilcoxon rank-sum test was used to compare BP distributions between classifications.

\section{Results}

One hundred patients (mean (SD) age 50.5 (12) years, 46\% male) were enrolled in the study. Fifty-four percent of patients were female, $20 \%$ were black African and $80 \%$ were of mixed ancestry. There were no significant differences in demographic characteristics of the patients who had therapeutic concentrations of the antihypertensive v. those who did not. Patients were taking a median of four antihypertensive medications. All patients were taking both an ACE inhibitor and amlodipine. Eighty percent were prescribed $10 \mathrm{mg}$ amlodipine daily, and $88 \%$ enalapril $10 \mathrm{mg}$ twice daily. The majority (90\%) of the patients administered their own medications, and $24 \%$ used pill boxes to assist with adherence. Ninety percent of patients knew the dosing schedule, $52 \%$ knew the names of the medications, $40 \%$ knew the dose, and $33 \%$ 
admitted to forgetting to take their medication regularly. There was no association between the answers to the questionnaire and amlodipine concentrations or ACE activity. All the patients were otherwise healthy with no clinical indication of malabsorption, and none was taking a medication known to decrease plasma concentrations of ACE inhibitors or amlodipine.

\section{ACE inhibitor results}

The median Z-FHL/HHL ratio for the entire cohort was 2.61 (IQR 1.47 - 3.12). Setting a ratio of $<1.75$ to indicate non-suppression of ACE, 26/97 (26.8\%) were not suppressed (Table 2). Three patients had missing data. There were significant differences in BP when suppression groups were compared based on medication concentration (Table 2). The median BPs were 142 (IQR 122 - 156)/82 (IQR 72 - 93) mmHg in the suppressed group and 163 (IQR $143-171$ )/102 (IQR $89-114$ ) mmHg in the nonsuppressed group; all $p$-values were statistically significant (Table 2). Only six patients who had suppressed ACE had BPs $>160 / 100 \mathrm{mmHg}$ (Fig. 1).

\section{Amlodipine results}

The median amlodipine concentration for the entire cohort was 13.6 (IQR 5.1 - 4.4) ng/mL. Amlodipine concentrations <2.5 ng/ $\mathrm{mL}$ were deemed to be undetectable and were measured in $20 / 100$ patients (20\%). Table 2 shows the BPs based on the patients' plasma concentrations. The dose of amlodipine did not affect serum concentrations, although only 14 patients were taking $5 \mathrm{mg}$ daily. The mean (SD) amlodipine concentration for the patients on $10 \mathrm{mg}$ was 15.6 (12.4) ng/mL and for those on $5 \mathrm{mg} 14.9$ (19.4) ng/mL. Four patients were taking $15 \mathrm{mg}$ amlodipine (concentration range 0 - $34 \mathrm{ng} / \mathrm{mL})$ and two patients $20 \mathrm{mg}(24.2 \mathrm{ng} / \mathrm{mL}$ and $25.5 \mathrm{ng} / \mathrm{mL})$. The median BPs were 140 (IQR 122 - 149)/84 (IQR 73 - 91) mmHg in those with concentrations $>7 \mathrm{ng} / \mathrm{mL}$ and 166 (IQR $160-187$ )/104 (IQR 96 - 117) $\mathrm{mmHg}$ in those with concentrations $<2.5 \mathrm{ng} / \mathrm{mL}$
( $p<0.001$ for differences between BPs in the two groups) (Table 2). Patients with in-between concentrations had BPs that were uncontrolled and between those of the other two groups (Table 2). Only two patients with BPs $>160 / 100 \mathrm{mmHg}$ had amlodipine concentrations $>7 \mathrm{ng} / \mathrm{mL}$ (Fig. 1).

\section{Discussion}

The major finding of this study was that $20 \%$ of patients had an undetectable amlodipine concentration and $27 \%$ had unsuppressed $\mathrm{ACE}$, and this was associated with significantly higher BPs compared with those with steady-state concentrations or suppressed ACE.

In addition, the patient questionnaires relating to administration of medication, use of pill boxes, knowing the dosing schedule, names and dosages of medications and admitting to missing medication did not correlate with therapeutic monitoring. We infer that the current method of assessing adherence via questioning at clinic visits may be inadequate and that the use of TDM may be a helpful adjunct.

Our findings suggest a paradigm shift for evaluating patients with apparent treatment resistance, and TDM should become an essential additional tool in evaluating these patients. It will not only identify patients with true resistance but also reduce the need for expensive tests looking for secondary causes, and reduce pill burden, the costs of medications and adverse events associated with polypharmacy. Furthermore, instead of focusing on over-investigating patients with perceived resistant hypertension, determining medication concentrations will focus attention on barriers to good adherence (e.g. fixed drug combinations or side-effects), preventing further costly investigations. ${ }^{[21]}$

Two recently published studies utilised TDM to determine antihypertensive medication adherence. ${ }^{[22,23]}$ However, both these studies determined TDM of multiple antihypertensives and used 24-hour ambulatory BP monitoring, which are not practical options in a resource-limited setting such as ours. Our study focused on the two most commonly prescribed antihypertensive medications

Table 1. Steady-state trough concentrations of amlodipine

\begin{tabular}{|c|c|c|}
\hline Amlodipine dose & $\begin{array}{l}\text { Steady state }(\mathrm{ng} / \mathrm{mL}) \text {, } \\
\text { mean }(\mathrm{SD})\end{array}$ & Population \\
\hline $15 \mathrm{mg}$ & $\mathrm{C}_{\min } 11.8(5.3)$ & White population, mean (SD) 25.8 (3.8) years ${ }^{[15]}$ \\
\hline $10 \mathrm{mg}$ & $\mathrm{C}_{\min } 15(4)$ & Asian population, mean (SD) 42.8 (10.7) years ${ }^{[16]}$ \\
\hline \multirow[t]{2}{*}{$5 \mathrm{mg}$} & $\mathrm{C}_{\min } 8.8(1)$ & White population, range 66 - 77 years $^{[17]}$ \\
\hline & $\mathrm{C}_{\min } 7(1)$ & White population, range $22-50$ years $^{[17]}$ \\
\hline
\end{tabular}

Table 2. Proportion of individuals considered suppressed/non-suppressed (ACE) or in steady state/undetectable (amlodipine) and BP, by group

\begin{tabular}{|c|c|c|c|}
\hline & $n(\%)$ & SBP (mmHg), median (IQR) & DBP (mmHg), median (IQR) \\
\hline Entire cohort $(N=100)$ & & $146(127-163)$ & $88(74-99)$ \\
\hline \multicolumn{4}{|l|}{ ACE inhibitor results $(N=97)$} \\
\hline Suppressed (Z-FHL/HHL ratio >1.76) & $71(73.2)$ & $142(122-156)$ & $82(72-93)$ \\
\hline Non-suppressed (Z-FHL/HHL ratio <1.75) & $26(26.8)$ & $163(143-171)$ & $102(89-114)$ \\
\hline$p$-value ${ }^{*}$ & & 0.0004 & $<0.0001$ \\
\hline \multicolumn{4}{|l|}{ Amlodipine results $(N=100)$} \\
\hline Steady state $(>7 \mathrm{ng} / \mathrm{mL})$ & $62(62.0)$ & $140(122-149)$ & $84(73-91)$ \\
\hline Undetectable $(<2.5 \mathrm{ng} / \mathrm{mL})$ & $20(20.0)$ & $166(160-187)$ & $104(96-117)$ \\
\hline Indeterminate $(2.5-7 \mathrm{ng} / \mathrm{mL})$ & $18(18.0)$ & $159(138-161)$ & $93(72-106)$ \\
\hline$p$-value ${ }^{\dagger}$ & & $<0.0001$ & $<0.0001$ \\
\hline
\end{tabular}




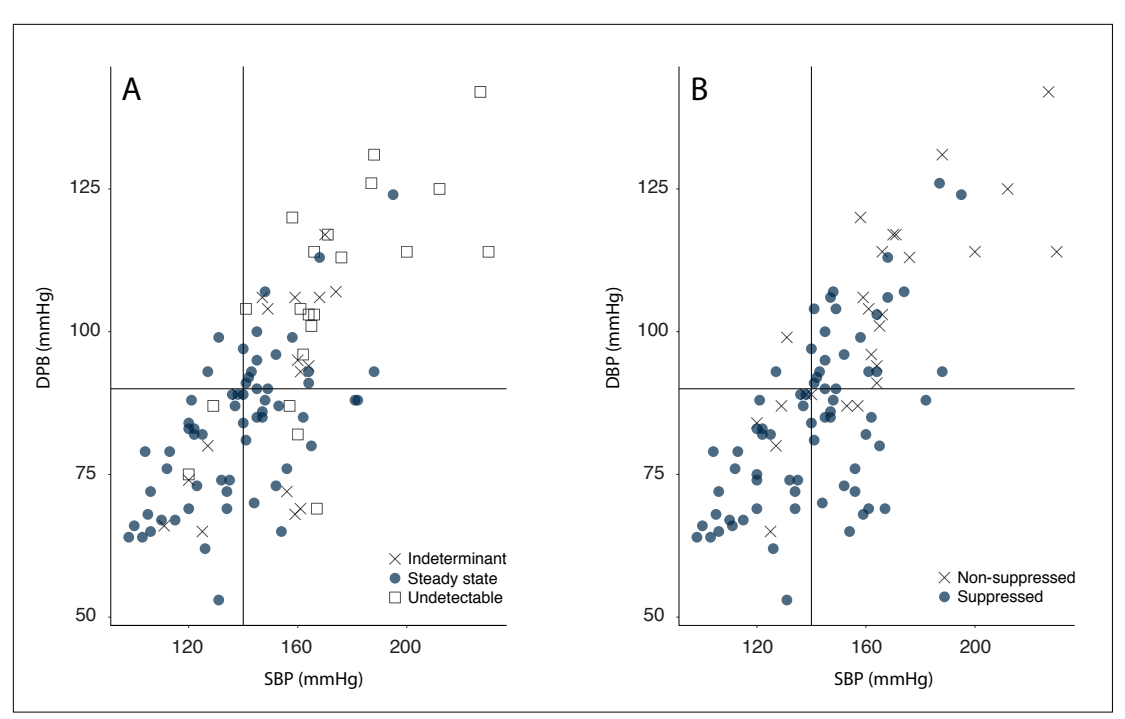

Fig. 1. Blood pressure according to adherence status for amlodipine levels (A) and Z-FHL/HHL (B). ( DBP = diastolic blood pressure; $S P B=$ systolic blood pressure. $)$

and suggests that the use of amlodipine TDM alone would help to detect complete lack of adherence and partial adherence in the majority of patients. The likely lack of adherence detected by amlodipine TDM in our follow-up patients was similar (32\% for completely and incompletely non-adherent) to the $37.9 \%$ reported. ${ }^{[23]}$ Quantified plasma concentrations of amlodipine allow monitoring of the degree of non-adherence, as opposed to purely detecting the drug's presence or not, or utilising the serum Z-FHL/HHL ratio (which can be used for all ACE inhibitors).

Finally, renal denervation has been advocated for the treatment of resistant hypertension. The Symplicity 1 and 2 studies $^{[24,25]}$ showed very promising results. However, the definitive Symplicity 3 trial, ${ }^{[26]}$ which was a randomised prospective blinded study, showed no BP benefit of renal denervation over good medical management. Unfortunately, definitive proof of medication adherence was not included as part of the inclusion criteria for these trials, possibly resulting in the confounding results. In our study, only a minority of patients met the inclusion criteria for renal denervation, namely office systolic BP $>160 \mathrm{mmHg}$ and fully adherent. In Leicester, the highest percentage of lack of adherence to all medications was in the group that was referred for renal denervation. ${ }^{[23]} \mathrm{A}$ recent analysis of patients who had stable medication adherence showed that renal denervation may be useful in cases with poor BP control despite documented concentrations of antihypertensives. ${ }^{[27]}$

\section{Study limitations}

There are some limitations in our study that warrant consideration: (i) we did not perform 24-hour BP monitoring to exclude pseudo-resistance; (ii) data relating to medication adherence analysis based on pharmacy refills were not available; and (iii) we did not assess reasons for lack of adherence. Nevertheless, automated clinic BP readings, as used in our study, have been shown to correlate with 24-hour monitoring and to reduce the white-coat effect. ${ }^{[28]}$

\section{Conclusions}

Our results show that patients with therapeutic plasma concentrations of amlodipine and suppression of ACE have better BP control than those who do not. TDM should become an essential tool in the evaluation of the patient with perceived resistant hypertension. This is of particular relevance to resource-limited environments, and may result in improved BP control, reduction in complications, reduced side-effects due to polypharmacy and reduction in healthcare costs. It will also allow the clinician to focus the consultation on the underlying reasons for non-adherence. Prospective studies are warranted to fully evaluate the potential for TDM in hypertensive patients in resourcelimited settings.

Acknowledgements. This work was presented in abstract format at the ISN World Congress of Nephrology and the 25th European Society of Hypertension Annual Meeting in 2015.

Author contributions. All the authors contributed to the writing of the article. ESWJ was involved in the design of the project, conducted the clinical research and wrote the article; ML designed the statistical analysis, performed the data analysis and prepared the results of the analysis for the article; MB and EHD were involved in the design of the research and review of the data; SC, SLUS, EDS and LW were involved in the design of the medication assays and assessment of the results; and BLR designed and supervised the project, analysed the data and supervised the writing of the article.

Funding. This project was funded by an unrestricted grant from the National Research Foundation of South Africa for rated researchers. Conflicts of interest. None.

1. Lim SS, Vos T, Flaxman AD, et al. A comparative risk assessment of burden of disease and injury attributable to 67 risk factors and risk factor clusters in 21 regions, $1990-2010$ : A systematic analysis for the Global Burden of Disease Study 2010. Lancet 2012;380(9859):2224-2260. https://doi. Study 2010. Lancet 2012;380(9859):22
org/10.1016/S0140-6736(12)61766-8

org $/ 10.1016 / \mathrm{S} 0140-6736(12) 61766-8$
2. Kearney PM, Whelton M, Reynolds K, Muntner P, Whelton

Kearney PM, Whelton M, Reynolds K, Muntner P, Whelton
PK, He J. Global burden of hypertension: Analysis of worldwide data. Lancet 2005;365(9455):217-223. https://doi.org/10.1016/ s0140-6736(05)70151-3

3. Rapsomaniki E, Timmis A, George J, et al. Blood pressure and incidence of twelve cardiovascular diseases: Lifetime risks, healthy life-years lost, and age-specific associations in 1.25 million people. Lancet 2014;383(9932):1899-1911. https://doi. org/10.1016/s0140-6736(14)60685-1

4. Persell SD. Prevalence of resistant hypertension in the United States, 2003-2008. Hypertension 2011;57(6):1076-1080. https:// States, 2003-2008. Hypertension 2011;57(6):1076-1080
doi.org/10.1161/HYPERTENSIONAHA.111.170308

5. Lewington S, Clark R, Qizilbash N, Peto R, Collins R, Lewington S, Clark R, Qizilbash N, Peto R, Collins R,
Prospective Studies Collaboration. Age-specific relevance of Prospective Studies Collaboration. Age-specific relevance of
usual blood pressure to vascular mortality: A meta-analysis of individual data for one million adults in 61 prospective studies. Lancet 2002;360(9349):1903-1913. https://doi.org/10.1016/ s0140-6736(02)11911-8

6. Smith WCS, Lee AJ, Crombie IK, Tunstall-Pedoe H. Control of blood pressure in Scotland: The rule of halves. BMJ 1990;300(6730): 981-983. https://doi.org/10.1136/bmj.300.6730.981

7. Burnier M. Medication adherence and persistence as the cornerstone of effective antihypertensive therapy. Am J Hypertens 2006;19(11):1190-1196. https://doi.org/10.1016/j. Hypertens 2006;19(11):11

8. Gosmanova EO, Lu JL, Streja E, Cushman WC, Kalantar-Zadeh Kosmanova EO, Lu JL, Streja E, Cushman WC, Kalantar-Zadeh
$\mathrm{K}$ Kovesdy CP. Association of medical treatment nonadherence $\mathrm{K}$, Kovesdy CP. Association of medical treatment nonadherence
with all-cause mortality in newly treated hypertensive US veterans. Hypertension 2014;64(5):951-957. https://doi. org/10.1161/hypertensionaha.114.03805

9. Vrijens B, Vincze G, Kristanto P, Urquhart J, Burnier M. Adherence to prescribed antihypertensive drug treatments: Longitudinal study of electronically compiled dosing histories. BMJ 2008;336(7653):11141117. https://doi.org/10.1136/bmj.39553.670231.25

10. Krousel-Wood MA, Muntner P, Islam T, Morisky DE, Webber LS. Barriers to and determinants of medication adherence in hypertension management: Perspective of the cohort study of hypertension management: Perspective of the cohort study of
medication adherence among older adults. Med Clin North Am 2009;93(3):753-769. https://doi.org/10.1016/j.mcna.2009.02.007

11. Sharma S, Joshi S, Mukherji S, Bala K, Tripathi CB. Therapeutic drug monitoring: Appropriateness and clinical utility in neuropsychiatry practice. Am J Ther 2009;16(1):11-16. https:// doi.org/10.1097/MJT.0b013e31817fd85f

12. Lie IA, Hoggen I, Samsonsen C, Brodtkorb E. Treatment nonadherence as a trigger for status epilepticus: An observational, retrospective study based on therapeutic drug monitoring. Epilepsy Res 2015;113:28-33. https://doi.org/10.1016/j.eplepsyres. 2015.03.007

13. Zoerner AA, Schroeder C, Kayacelebi AA, et al. A validated, rapid UPLC-MS/MS method for simultaneous ivabradine, reboxetine, and metoprolol analysis in human plasma and its reboxetine, and metoprolol analysis in human plasma and its
application to clinical trial samples. J Chromatogr B Analyt application to clinical trial samples. J Chromatogr B Analyt
Technol Biomed Life Sci 2013;927:105-111. https://doi. org/10.1016/j.jchromb.2013.01.016

14. Stangier J, Su CPF. Pharmacokinetics of repeated oral doses of amlodipine and amlodipine plus telmisartan in healthy volunteers. J Clin Pharmacol 2000;40(12):1347-1354. https:// doi.org/10.1177/009127000004001206

15. Faulkner JK, McGibney D, Chasseaud LF, Perry JL, Taylor IW. The pharmacokinetics of amlodipine in healthy volunteers after 
single intravenous and oral doses and after 14 repeated oral doses given once daily. Br J Clin Pharmacol 1986;22(1):21-25. https://doi.org/10.1111/j.1365-2125.1986.tb02874.x

16. Bhad P, Ayalasomayajula S, Karan R, et al. Evaluation of pharmacokinetic interactions between amlodipine, valsartan, and hydrochlorothiazide in patients with hypertension. J Clin Pharmacol 2011;51(6):933-942. https://doi.org/10.1177/0091270010376963

17. Leenen FH, Coletta E. Pharmacokinetic and antihypertensive profile of amlodipine and felodipine-ER in younger versus older patients with hypertension. J Cardiovasc Pharmacol 2010;56(6):669-675. https://doi.

18. Danilov SM, Balyasnikova IV, Albrecht RF, Kost OA. Simultaneous determination of ACE activity with 2 substrates provides information on the status of somatic ACE and allows detection of inhibitors in human blood. J Cardiovasc Pharmacol 2008;52(1):90-103. https://doi.org/10.1097/ FJC.0b013e31817fd $3 \mathrm{~b}$

19. Seedat YK, Rayner BL, Veriava Y. South African hypertension practice guideline 2014. Cardiovasc J Afr 2014;25(6):288-294. https://doi.org/10.5830/CVJA-2014-062

20. Schwager SL, Carmona AK, Sturrock ED. A high-throughput fluorimetric assay for angiotensin I-converting enzyme. Nat Protoc 2006;1(4):1961-1964. https://doi.org/10.1038/nprot.2006.305

21. Hess G, Hill JD, Lau H, Dastani H, Chaudhari P. Medication utilization patterns and hypertensionrelated expenditures among patients who were switched from fixed-dose to free-combination antihypertensive therapy. Pharm Ther 2008;33(11):652-666.

22. Strauch B, Petrak O, Zelinka T, et al. Precise assessment of noncompliance with the antihypertensive therapy in patients with resistant hypertension using toxicological serum analysis. J Hypertens 2013;31(12):2455-2461. https://doi.org/10.1097/HJH.0b013e3283652c61
23. Tomaszewski M, White C, Patel P, et al. High rates of non-adherence to antihypertensive treatment revealed by high-performance liquid chromatography-tandem mass spectrometry (HP LC-MS/MS) urine analysis. Heart 2014;100(11):855-861. https://doi.org/10.1136/heartjnl-2013-305063

24. Krum H, Schlaich MP, Sobotka PA, et al. Percutaneous renal denervation in patients with treatmentresistant hypertension: Final 3-year report of the Symplicity HTN-1 study. Lancet 2014;383(9917):622629. https://doi.org/10.1016/s0140-6736(13)62192-3

25. Esler MD, Krum H, Sobotka PA, Schlaich MP, Schmieder RE, Bohm M. Renal sympathetic denervation in patients with treatment-resistant hypertension (the Symplicity HTN-2 Trial): A randomised controlled trial. Lancet 2010;376(9756):1903-1909. https://doi.org/10.1016/s0140-6736(10)62039-9 26. Bhatt DL, Kandzari DE, O'Neill WW, et al. A controlled trial of renal denervation for resistan hypertension. N Engl J Med 2014;370(15):1393-1401. https://doi.org/10.1056/NEJMoa1402670

27. De Jager RL, de Beus E, Beeftink MMA, et al. Impact of medication adherence on the effect of rena denervation: The SYMPATHY Trial. Hypertension 2017;69(4):678-684. https://doi.org/10.1161/ HYPERTENSIONAHA.116.08818

28. Van der Wel MC, Buunk IE, van Weel C, Thien TA, Bakx JC. A novel approach to office blood pressure measurement: 30-minute office blood pressure vs daytime ambulatory blood pressure. Ann Fam Med 2011;9(2):128-135. https://doi.org/10.1370/afm.1211

Accepted 22 May 2017 